Ilseana, $\boldsymbol{R}$. rubescens, $\boldsymbol{R}$. laxifolia Borb., die Kelchblätter sind verhältnissmässig kurz, die äusseren davon besitzen nur wenige und nur sehr schmale Zipfel. Der Kelch breitet sich nach Kmet auf der halbreifen Scheinfrucht sternförmig aus oder richtet sich gänzlich auf und bleibt so lange wie bei $R$. Ilseana auf der Frucht. Auch der weisswollige Kopf der Griffel, die kurzen, manchmal fast fehlenden und bereiften Pedunculi, die reifen Scheinfrüchte, sowie die schlanken, dünnen, sehr stark gekrümmten, hie und da fast quirlig stehenden Stacheln stimmen mit jenen der $R$. Ilseana überein.

$\boldsymbol{R}$. Pokornyana Kmet $\boldsymbol{R}$. imponenti Rip. apud Borb. Mon. Rosar. proxima, sed differt aculeis gracilibus hinc et inde fere verticillatis, valde uncinatis, foliolorum dentibus minus compositis, in foliis inferioribus fere biserratis, in superioribus simplicibus, sepalis abbreviatis lacinitis paucis, angustissimisque receptaculis et fructibus minoribus ovoideis, breviter pedicellatis, nonnullis eorum subsessilibus, a $R$. Graveti praeterea foliolis haud simpliciter serratis.

Wenn man $\boldsymbol{R}$. Poleornyana nach meiner Monogr. Rosar. bestimmen will, so kommt man p. $445 \mathrm{zu} \mathrm{Nr} .29$, und da muss man sie mit folgender Diagnose einreihen.

Aculeis gracilibus, hinc et inde fere verticillatis, valde uncinatis; foliolis obovatis, basi breviter cmeatis, in foliis inferioribus fere biserratis aut omnino biserratis, in superioribus simpliciter serratis et saepe lanceolatis, supra obscure viridibus, subtus pallidioribus; pedunculis aggregatis brevibus, bracteis superatis glaucis, interdum brevissimis, sepalis abbreviatis parce et anguste laciniatis, petalis intense roseis, fructu ovoideo.

Rosa Pokornyana ist jedenfalls ein interessantes Glied der "Montanae", durch die Form der Stacheln ist sie auch mit $R$. incana und $\boldsymbol{R}$. Kmetiana Borb. verwandt, und zeichnen sich also durch diese Form der Stacheln mehrere Rosenformen der Selmeczer Flora aus.

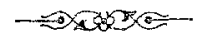

\title{
Flora von Meran im Mai a. c.
}

\section{Von Prof. Dr. Entleutner.}

Angenebm wandelt sich's unter dem Schatten von Nuss-, Kastanien- und Maulbeerbäumen an weinberggrünen Hügeln, an hochhalmigen Getreidefeldern und frisch gemähten Wiesen vorbei. Ueber herrlichen Rosenbüschen und duftenden Blumenkronen schwebt unser schönster Alpenfalter, Parnasius Apollo. Mit ihm zieht es auch uns hinauf zu den maigrünen Almen. Ja steigen wir hinauf zur $2226 \mathrm{M}$. hohen Muttspitze.

Schon am Gratscher Fahrweg notiren wir: Centaurea nigrescens, Rumex obtusifolius et crispus, Sedum dasyphyllum, Cardamine impatiens. 
Auf dem nun über Durnstein nach St. Peter hinaufführenden Wege blühen an den mit Quercus pubescens Willd. bewachsonen Abhängen: Tunica saxifraga Scop., Linaria genistifolia D C., Phyteuma Michelii Bertol, Helianthemum Fumana Mill., Orobanche Hederae Vauch, Epithymum DC. und arenaria Borkh., Allium sphaerocephalum und fallax Dan., Campanula spicata und Rapunculus, Sempervivum arachnoideum, Colutea arborescens, Anthericum Liliago, Specularia Speculum DC., Papaver dubium, Dianthus silvestris Wulf., Silene Armeria, Vicia lutea, Galium lucidum All., Potentilla recta, Cynanchum laawm Bartl., Luzula nivea DC., Andropogon Ischaemum, Koeleria cristata Pers. und Festuca myuros Ehrh.

Vor Schloss Tirol. steigen wir durch ein Fichtenwäldchen, in dem eine unserer schönsten Orchideen (Limodorum abortivum $\mathrm{Sw}$.) blüht, neben den Erdpyramiden steil aufwärts. An einem Bauernhofe vorbei geht es über Wiesen, auf denen wir Orchis Morio, maculata und variegata All., Platanthera bifolia Rich., Aquilegia atrata Koch, Gentiana excisa Koch und Luzula albida DC. $\beta$. rubella erblicken.

Wieder durch Nadelwald emporsteigend, in dem der Kukuksruf erschallt, und das muntere Eichhörnchen blitzschnell von Ast zu Ast sich schwingt, kommen wir zu einer von einem Bächlein durchrieselten Schlucht. Hier finden wir: Pinguicula vulgaris $\beta$. grandifora, Trollius europaeus, Saxifraga Aizoon Jeq. und aspera. Und abermals durch Wald führt uns der Weg hinauf zum Hof des Klaus, vor dem der Kirschbaum jetzt erst blüht, während wir uns im Thale unten schon der reifen Frucht orfreuen.

Die nun folgenden Bergwiesen sind überkleidet mit blühenden Anemonen ( $A$. alpina $\beta$. sulphurea und $A$. vernalis) und Orchideen (Orchis sambucina mit der Var. $\beta$. purpurea und O. ustulata). Auch Saxifraga Clusii Gouan, Silene rupestris, Aster alpinus, Alchemilla pubescens M. B., Homogyne alpina Cass., Petasites albus Gaertn., Potentilla aurea und Cerastium arvense $\gamma$. suffruticosum finden wir hier. Aus den Felsspalten winkt uns die herrliche Primula villosa Jacq., und da und dort überrascht uns die Primula longifolia. In dichten Polstern überdeckt Arctostaphylos offic. und auf dem Grat anch die niedliche Azalea procumbens das Schiefergestein. Auf der Spitze blühen auch schon Geum montanum und Soldanella pusilla Baumgartn.

Zufrieden mit dem Resultate unserer Excursion steigen wir wieder ins Etschthal hinab, in dem sich noch so manche Blüthenknospe erschlossen hat. So sehen wir auf den Mösern, Kiesbänken und in den Altwassern der Etsch: Nasturtium officinale, Euphorbia Gerardiana Jacq., Plantago altissima, Typha minima, Polygala amara, Hieracium praealtum Koch, Batrachium paucistamineum Tausch., Myricaria germanica, Carex Oederi, Glyceria fluitans R. Br., Alopecurus fulvus Sm., Agrostis alba, Iris Pseudacorus. 
Bei Katzenstein: Lilium bulbiferum und Martagon, Brachypodium pinnatum Beauv., Digitalis lutea.

Im Naifthal blühen: Arabis alpina, Thalictrum foetidum und bei den Quadrathöfen: Atragene alpina, Sambucus racemosa, Lonicera nigra, Gentiana verna und aestiva R. et Sch.

Bei Katzenstein und Burgstall findet man: Dictamnus Fraxinella Pers., Cephatanthera ensifolia Rich., Veronica urticifolia, Orobus variegatus Ten., Centranthus ruber DC. und Bryonia dioica Jeq.

Gehen wir über das von Sümpfon umgebene Plans nach Schloss Dornsberg mit seiner Zugbrücke, der hohen Halle und dem sehenswerthen, mit Gallerien umgebenen Hof, so können wir auf unserem Wege notiren: Pedicularis palustris, Menyanthes trifoliata, Diplotaxis tenuifolia DC., Orchis incarnata und militaris, Primula farinosa, Scirpus compressus, Saliw pentandra.

Gymnadenia odoratissima Rich. und Crepis paludosa Moench. bemerken wir bei St. Felix; Aristolochia Clematitis, Avena puhescens und Brachypodium silvaticum bei Obermais; Medicago minima, Dianthus atrorubens All., Silene Otites Sm., Jasione montana und Gnaphalium luteo-album auf dem Küchelberg.

Endlich gehen wir noch in die Masulschlucht bei Verdins, denn dort glüht in schwellenden Büscheln die duftende Alpenrose (Rhododendron ferrugineum).

\section{Flora des Etna.}

\section{Von Prof. P. Gabriel Strobl.}

(Fortsetzung.)

720. Lycopsis variegata L. *Bert., bullata Cyr. *Raf. II, Anchusa varieg. Lehm. *Guss. Prodr., *Syn. et *Herb.! An steinigen, sandigen Weg- und Feldrändern, auf Lavaströmen, zwischen Gebüsch und in lichten Wäldern $\left(0-5000^{\circ}\right)$, besonders in der höheren Tiefregion sehr verbreitet: Um Bronte und Valetto (!, Guss. Prodr., Syn. et Herb.!), Acireale (Guss. Syn. Add.), Pedara, Vallone di Ulli (Herb. Tornab.!), aus dem Valle del Bue von Cosentini erhalten (Bert.), sehr gemein von Nicolosi zum Monte Nocilla, Bosco di Malpasso, zur Casa del Bosco, aber auch hänfig zwischen Nicolosi und Catania! März-Juni. $\odot$.

NB. orientalis *Raf. I kenne ich nicht, anch Bert. und Guss. erwähnen sie nicht.

721. Symphytum Zeyeri Schmp. Guss. Syn. et *Herb.!, bulbosum Guss. Prodr., non Schimp. An Zäunen, buschigen und cultivirten Orten der Tiefregion bis 3000': Am Etna und ob Giarre (Cosent. in Herb. Guss.!), Catania, Milo (Herb. Tornab.!). März, April. 2 . NB. officinale *Raf. II. dürfte wohl auch hieher gehören. 